\title{
COOPERATIVE POSITIONING OR UAV SWARMS BY FUSING IMU/UWB/GPS WITH FEDERAL KALMAN FILTER
}

\author{
Rubin Zou \\ Nashua High School South, Nashua, NH, USA
}

\begin{abstract}
A wide variety of sensors have been used to attempt to most accurately calculate the positions of UAV drones in swarms. Global Positioning System (GPS), Inertial Measurement Unit (IMU), and Ultra-Wideband (UWB) are the most common and the most reliable sensors that are used. This proposed fusion method uses a novel Double Kalman Filter method to combine the data from these 3 sensors to most accurately achieve cooperative positioning. In the simulation, we used 30 UAVs as a swarms to verify the validity of the method. As the results shown, our method outperformed several other methods.
\end{abstract}

Key words: UAV Swarms, IMU, UWB, GPS, Federal Kalman, Filter

Cite this Article: Rubin Zou, Cooperative Positioning or UAV Swarms by Fusing IMU/UWB/GPS with Federal Kalman Filter, International Journal of Computer Engineering and Technology 10(5), 2019, pp. 38-46.

http://iaeme.com/Home/issue/IJCET?Volume=10\&Issue $=5$

\section{INTRODUCTION}

In the near future, our airspace will be populated by swarms of aerial robots. These autonomous Unmanned Aerial Vehicles (UAVs) are emerging as a new technology that provides highly-reconfigurable, on-demand, distributed intelligent autonomous systems, drastically improves efficiency and capability for many different tasks, including tracking, inspection, and transporting systems. In any applications, autonomous aerial swarms are expected to be more capable than a single large vehicle, offering significantly enhanced flexibility (adaptability, scalability, and maintainability) and robustness (reliability, survivability, and fault-tolerance) [1].

In order to control a drone swarm, we must be able to precisely and accurately locate the position of each drone. Many different sensors have been applied to attempt to do this. Global Positioning Systems (GPS) provide absolute longitudinal and latitudinal information [1]. The advantages of using GPS is that can provide position information almost anywhere on the globe. GPS also recalculates position on every call, and does not use the previous estimate in the new calculation. This means that it will not degrade over time. The disadvantages of GPS is that it is quite inaccurate. The GPS accuracy will be affected by a number of factors, such as noise in the radio signal, atmospheric conditions, satellite positions and natural barriers to 
the signal. The signal noise results from signal disruption near the receiver or different signals in the same frequency, and creates errors from 1 meter to 10 meters. Some high objects such as mountains or buildings that are between the satellite and the receiver can also produce errors, and the bias could be up to 30 meters [2]. A solution to this problem is to use stationary receivers to adjust the GPS readings and eliminate errors. An example of this is Differential GPS (DGPS), which can improve accuracy to 3 feet or better [2]. GPS receivers also can feature various deficiencies during fast movement, during the start phase of the operation, or in the case of a strong multipath effect [3]. Another issue with GPS is that obstructions greatly reduce its capability, so it is practically useless indoors. Inertial Measurement Units (IMU), which is within many Inertial Navigation Systems (INS), are devices that combine multiple sensors, including accelerometers, gyroscopes, altimeters, etc, to calculate information about position, velocity, acceleration, and orientation. The advantages of IMU is that it can be built into the drone itself, and it doesn't require any signals to run, meaning it will always be able to provide information given that the IMU is functional. The disadvantages of IMU is that, while in short periods of time it can provide very accurate information, the errors will add up over long periods of time, making it unreliable. Radio signals have also been used to find distances between nodes. These include Ultra Wideband (UWB), RF propagation, etc. In theory, UWB is very resistant to multipath, provided that the line-of-sight signal is detected in the first place [4]. This makes it a better option than regular radios because smaller radios' signal strength at short ranges is subject to unpredictable variation due to fading, multipath, and interferences; therefore, it does not correlate directly with distance [7].

APIT requires a heterogeneous network of sensing devices where a small percentage of these devices (percentages vary depending on network and node density) are equipped with high-powered transmitters and location information obtained via GPS or some other mechanism. A perfect Point-In-Triangulation Test (PIT) is impossible, but a very accurate approximation is possible[5]. Bounding Box method for localizing nodes in an ad hoc communication network takes each node and creates a signal bounding box around it. Then, to calculate the position of a node, signals are sent out from the target node to other nodes. If the signal is received, the neighboring nodes sends back its position, and if no signal is received, there is no return signal. After all known signals are received, the estimated position is calculated as the intersection of the neighboring bounding boxes [6]. Centroid method uses an RF-based localization method in which the target node localizes itself with high confidence (under an idealized radio model) to the centroid of a set of proximate reference points using a connectivity metric. The advantage of this method is that it does not use GPS, which makes it easy to use. The approach produced decent results, in outdoor environments, the model correlated with reality at 87 percent. The framework of the UWB method consists of an anchor robot with three UWB sensors and a tag robot with a single UWB sensor. The anchor robot utilizes the three UWB sensors as a localization infrastructure and estimates the tag robot's location by using its on-board sensing and computational capabilities solely, without explicit inter-robot communication. The method's framework utilizes a dual Monte-Carlo localization (MCL) algorithm. This method outperformed their standard particle filter and extended Kalman filter algorithm [8]. To deal with the contradiction between positioning accuracy and computational complexity, statistical linear regression is used to design the posterior linearization method of measurement model, and the linearized measurement model is used on the algorithm framework of cooperative positioning which is based on belief propagation. Their simulation results showed that this method, compared with the noncooperative case, improves the positioning accuracy of the follower UAVs in $\mathrm{X}, \mathrm{Y}$ and $\mathrm{Z}$ directions (earth-centered earth-fixed coordinate system, ECEF) by 8.9, 13.9, 17.3 times respectively [9]. RSS and MSM method for large scale distributed localization utilizes 
received signal strength (RSS) and a mass-spring model (MSM). In their simulation, an average error of up to $7.66 \mathrm{~m}$ could be achieved in a field of $100 \mathrm{~m}$ by $100 \mathrm{~m}$.

To accurately estimate the position of each UAV drone in a swarm, a method to achieve cooperative positioning of the drones is necessary. The most common sensors on modern drones are GPS, IMU/INS, and some form of radio signal (in our case will be UWB). A fusion method for these sensors must be implemented to control a drone swarm. In this paper, I will demonstrate a method using a Double Kalman Filter method the fuse GPS, IMU, and UWB together to find the most accurate estimation of the positions of drones in a drone swarm.

\section{PROBLEM FORMULATION}

IMU/UWB/GPS fusion positioning model : Dynamic model and observation model

Variables:

$t$ - time

$x_{t}-$ signal value at time $\mathrm{t}$

$z_{t}$ - measurement value at time $\mathrm{t}$

$u_{t}$ - control signal at time $\mathrm{t}$

$w_{t}$ - process noise at time $\mathrm{t}$

$v_{t}$ - measurement noise at time $\mathrm{t}$

$A$ - state transition matrix

$B$ - control matrix

$H$ - observation matrix

$m_{t}-$ measurement

$U_{t}-$ control signal vector at time $\mathrm{t}$

$Q$ - measurement noise

$R$ - environment noise

$A^{T}-\mathrm{A}$ transpose matrix

$H^{T}-\mathrm{H}$ transpose matrix

$X_{t}^{-}$- a priori estimation of position at time $\mathrm{t}$

$X_{t}$ - a posteriori estimation of position at time $\mathrm{t}$

$P_{t}^{-}-$a priori error covariance of position at time $\mathrm{t}$

$P_{t}$ - a posteriori error covariance of position at time $\mathrm{t}$

$\dot{X}_{t}^{i m u}-$ movement vector from IMU at time $\mathrm{t}$

$Q_{t}^{i m u}$ - IMU noise at time $\mathrm{t}$

$K_{t}$ - Kalman Gain at time t

$R_{t}^{g p s}-$ GPS noise at time $\mathrm{t}$

$X_{t}^{i}$ - a priori estimation of the position of drone $\mathrm{i}$ at time $\mathrm{t}$

$X_{t}^{i^{*}}$ - a posteriori estimation of the position of drone $i$ at time $t$

$P_{t}^{i}$ - a priori error covariance of the position of drone $\mathrm{i}$ at time $\mathrm{t}$

$P_{t}^{1^{*}}$ - a posteriori error covariance of the position of drone $i$ at time $t$

$K_{t}^{i}$ - Kalman Gain of drone $\mathrm{i}$ at time $\mathrm{t}$ 
$X_{u w b}^{(i, j)}-\mathrm{UWB}$ measured distance from drone $\mathrm{i}$ to drone $\mathrm{j}$ based on the UWB radio of drone $\mathrm{i}$ at time $\mathrm{t}$

$R_{t}^{u w b^{(1,2)}}-$ UWB error of the distance measurement from drone $\mathrm{i}$ to drone $\mathrm{j}$ based on the UWB radio of drone $i$ at time $t$

A system dynamics model is a dynamic method (based on changes in time) to model realworld phenomenon using mathematical equations. The basic mathematical structure of a system dynamics model consists of coupled, nonlinear, first-order differential or integral equations [11]. The model bases itself off of the previous estimates. The system dynamics model models the IMU output. The IMU model is as follows:

$$
X_{t}=A X_{t-1}+B \dot{X}_{t}^{i m u}+w_{t}^{i m u}
$$

The equation can be generalized in a matrix and vector form as:

$$
x_{t}=A x_{t-1}+B u_{t}+w_{t-1}
$$

An observation model is a model based on observed phenomenon. The observed phenomenon is not based off of previous estimates, which means at each point in time, a new value is generated.

The observation model models the GPS and UWB output. The GPS model is as follows:

$$
X_{t}=H X_{t}+w_{t}^{g p s}
$$

The UWB model is as follows

$$
d_{t}=H d_{t}+w_{t}^{u w b}
$$

These equations can be generalized as

$$
z_{t}=H x_{t}+v_{t-1}
$$

\section{FEDERAL KALMAN FILTER METHOD FOR IMU/UWB/GPS}

\subsection{Federal Kalman filter}

Fusion of the dynamics model and the observation models can be achieved using out first Kalman filter method. Kalman Filters use estimations and estimation errors to calculate the most probable position of a node based off of dynamic data and observable data. The first step of this Kalman Filter method is to estimate the new position, the a priori estimation, based off of the dynamic model. This is done using previous data and the new IMU data. First, the a priori estimate is as follows:

$$
X_{t}^{-}=A X_{t-1}+B U_{t}=A X_{t-1}+\dot{X}_{t}^{i m u}
$$

and the a priori error covariance is:

$$
P_{t}^{-}=A P_{t-1} A^{T}+Q=A P_{t-1} A^{T}+Q_{t}^{i m u}
$$

The next step is to calculate the Kalman Gain. The Kalman Gain uses the a priori error covariance, as well as the error of the observable GPS data, to calculate how much of the a priori estimation to trust, and how much of the GPS data to trust.The Kalman Gain can be calculated as follows:

$$
K_{t}=\frac{P_{t}^{-} H^{T}}{H P_{t}^{-} H^{T}+R}=\frac{\left(A P_{t-1} A^{T}+Q_{t}^{i m u}\right) H^{T}}{H\left(A P_{t-1} A^{T}+Q_{t}^{i m u}\right) H^{T}+R_{t}^{g p s}}
$$

The last step of this Kalman Filter is to calculate a final estimated position, the a posteriori estimation, using the Kalman Gain, the a priori estimation, and the GPS data. Then, the a posteriori estimation:

$$
X_{t}=X_{t}^{-}+K_{t}\left(Z_{t}-X_{t}^{-}\right)
$$




$$
=A X_{t-1}+\dot{X}_{t}^{i m u}+\frac{\left(A P_{t-1} A^{T}+Q_{t}^{i m u}\right) H^{T}}{H\left(A P_{t-1} A^{T}+Q_{t}^{i m u}\right) H^{T}+R_{t}^{g p s}}\left(Z_{t}^{g p s}-\left(A X_{t-1}+\dot{X}_{t}^{i m u}\right)\right)
$$

and the a posteriori error covariance:

$$
P_{t}=\left(I-K_{t}\right) P_{t}^{-}=\left(1-\frac{\left(A P_{t-1} A^{T}+Q_{t}^{i m u}\right) H^{T}}{H\left(A P_{t-1} A^{T}+Q_{t}^{i m u}\right) H^{T}+R_{t}^{g p s}}\right)\left(A P_{t-1} A^{T}+Q_{t}^{i m u}\right)
$$

In order to integrate UWB data, we can use a second Kalman Filter method. This Kalman Filter will calculate based on data from pairs of drones.

The following data are the posteriori estimations and error covariances of each drone, calculated above. Essentially, our previous a posteriori estimations become the new a priori estimations. The state of ith drone is expressed as $X_{t}^{i}, P_{t}^{i}$. There are two Kalman Gains for this pair of drones, the Kalman Gain for the first drone, and the Kalman Gain for the second Drone. These Kalman Gains are calculated a little differently, because three values must be involved, the error covariance of the first drone, the error covariance of the second drone, and the error of the UWB data taken between the drones. The Kalman Gain for the first drone is calculated by multiplying the Kalman Gain between the two drones, and the Kalman Gain between the estimations and the UWB data. Calculating the Kalman Gain for the first drone:

$$
\begin{gathered}
K_{t}^{1}=\left(\frac{P_{t}^{1} H^{T}}{H P_{t}^{1} H^{T}+H P_{t}^{2} H^{T}}\right)\left(\frac{P_{t}^{1} H^{T}+P_{t}^{2} H^{T}}{H P_{t}^{1} H^{T}+H P_{t}^{2} H^{T}+R_{t}^{u w b^{(1,2)}}}\right) \\
=\left(\frac{P_{t}^{1} H^{T}}{H P_{t}^{1} H^{T}+H P_{t}^{2} H^{T}+R_{t}^{u w b^{(1,2)}}}\right)
\end{gathered}
$$

Is this case, the larger $P_{t}^{2}$ and $R_{t}^{u w b^{(1,2)}}$ are, the less we trust the UWB data, and the smaller $P_{t}^{2}$ and $R_{t}^{u w b^{(1,2)}}$ are, the more we trust the UWB data. Similarly, we calculate the Kalman Gain for the second drone:

$$
\begin{gathered}
K_{t}^{2}=\left(\frac{P_{t}^{2} H^{T}}{H P_{t}^{1} H^{T}+H P_{t}^{2} H^{T}}\right)\left(\frac{P_{t}^{1} H^{T}+P_{t}^{2} H^{T}}{H P_{t}^{1} H^{T}+H P_{t}^{2} H^{T}+R_{t}^{u w b^{(1,2)}}}\right) \\
=\left(\frac{P_{t}^{2} H^{T}}{H P_{t}^{1} H^{T}+H P_{t}^{2} H^{T}+R_{t}^{u w b^{(2,1)}}}\right)
\end{gathered}
$$

Then, using these new Kalman Gains, we can calculate new estimations of the positions of the drones, and new error covariances. The new estimations:

$$
\begin{aligned}
& X_{t}^{1^{*}}=X_{t}^{1}+\left(\frac{P_{t}^{1} H^{T}}{H P_{t}^{1} H^{T}+H P_{t}^{2} H^{T}+R_{t}^{u w b^{(1,2)}}}\right)\left(\left(X_{t}^{2}-X_{u w b}^{(1,2)}\right)-X_{t}^{1}\right) \\
& X_{t}^{2^{*}}=X_{t}^{2}+\left(\frac{P_{t}^{2} H^{T}}{H P_{t}^{1} H^{T}+H P_{t}^{2} H^{T}+R_{t}^{u w b^{(2,1)}}}\right)\left(\left(X_{t}^{1}+X_{u w b}^{(2,1)}\right)-X_{t}^{2}\right)
\end{aligned}
$$

And the new error covariances:

$$
\begin{aligned}
& P_{t}^{1^{*}}=\left(1-\left(\frac{P_{t}^{1} H^{T}}{H P_{t}^{1} H^{T}+H P_{t}^{2} H^{T}+R_{t}^{u w b^{(1,2)}}}\right)\right) P_{t}^{1} \\
& P_{t}^{2^{*}}=\left(1-\left(\frac{P_{t}^{2} H^{T}}{H P_{t}^{1} H^{T}+H P_{t}^{2} H^{T}+R_{t}^{u w b^{(2,1)}}}\right)\right) P_{t}^{2}
\end{aligned}
$$




\subsection{Cooperative positioning with IMU/UWB/GPS}

The model for the Kalman Filter is basically an infinite loop, where new estimations are made for every increment of time. Let's say time is currently $t$. The loop begins by finding the IMU estimations, which are based off of previous values (from t-1) and current IMU data. The state matrix model for the IMU is as follows:

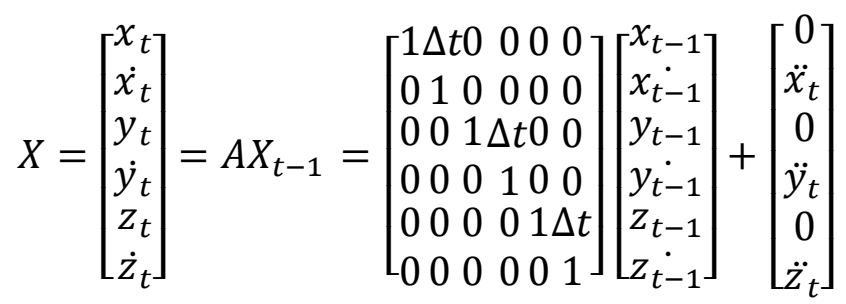

Next, GPS is fused in by using the first Kalman Filter method. We can equate position by multiplying our different estimations and sensor values by different forms of the Kalman Filter, as described in the equations below.

$$
\begin{gathered}
x_{t}=x_{t}^{-}+K_{t}\left(m_{t}-x_{t}^{-}\right)=K_{t} m_{t}-\left(K_{t}-1\right) x_{t}^{-}=K_{t} m_{t}-\left(K_{t}-1\right)\left(x_{t-1}+\Delta t * \dot{x_{t}}\right) \\
=K_{t} m_{t}-\left(K_{t} x_{t-1}+K_{t} * \Delta t * \dot{x_{t}}-x_{t-1}-\Delta t * \dot{x_{t}}\right)
\end{gathered}
$$

Then, the matrix after including GPS is:

$$
X=\left[\begin{array}{c}
x_{t-1} \\
x_{t}^{i m u} \\
x_{t}^{g p s} \\
y_{t-1} \\
y_{t}^{i m u} \\
x_{t}^{g p s} \\
z_{t-1}^{\text {mpu }} \\
z_{t}^{i \dot{m} u} \\
x_{t}^{g p s}
\end{array}\right]\left[\begin{array}{ccccccccr}
1-K_{t}\left(1-K_{t}\right) \Delta t K_{t} & 0 & 0 & 0 & 0 & 0 & 0 \\
0 & 1 & 0 & 0 & 0 & 0 & 0 & 0 & 0 \\
0 & 0 & 1 & 0 & 0 & 0 & 0 & 0 & 0 \\
0 & 0 & 0 & -K_{t}\left(1-K_{t}\right) \Delta t K_{t} & 0 & 0 & 0 \\
0 & 0 & 0 & 0 & 1 & 0 & 0 & 0 & 0 \\
0 & 0 & 0 & 0 & 0 & 1 & 0 & 0 & 0 \\
0 & 0 & 0 & 0 & 0 & 0 & -K_{t}\left(1-K_{t}\right) \Delta t K_{t} \\
0 & 0 & 0 & 0 & 0 & 0 & 0 & 1 & 0 \\
0 & 0 & 0 & 0 & 0 & 0 & 0 & 0 & 1
\end{array}\right]
$$

The last step is to fuse UWB into the estimations using the second Kalman Filter method. The matrix for comparing 2 drones:

$$
\left[\begin{array}{c}
X_{t}^{1} \\
P_{t}^{1} \\
X_{u w b}^{(1,2)} \\
X_{t}^{2} \\
P_{t}^{2}
\end{array}\right]\left[\begin{array}{ccccc}
\left(1-K_{t}\right) & 0 & -K_{t} & K_{t} & 0 \\
0 & \left(1-K_{t}\right) & 0 & 0 & 0 \\
0 & 0 & 1 & 0 & 0 \\
K_{t} & 0 & -K_{t}\left(1-K_{t}\right) & 0 \\
0 & 0 & 0 & 0 & \left(1-K_{t}\right)
\end{array}\right]
$$

We repeat the second Kalman Filter process for every UWB signal available at that time. If a UWB signal between a certain pair of drones is not available at a specific time, we skip the second Kalman Filter for that specific pair at that specific time. Then, when time increments to $\mathrm{t}+1$, we restart the loop, and our final estimations from time $t$ because our new "previous values". The proposed algorithms architecture is shown as figure 1. 


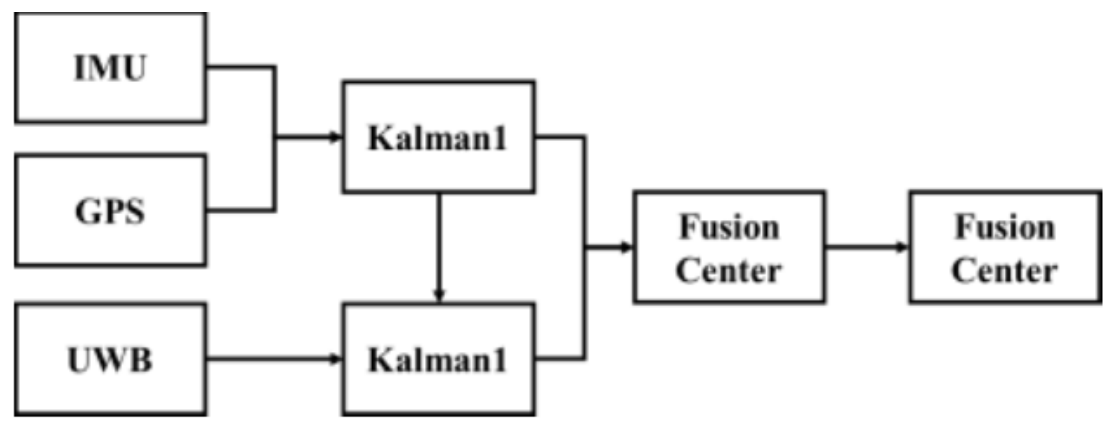

Figure 1 Algorithms architecture

\section{SIMULATION AND ANALYSIS}

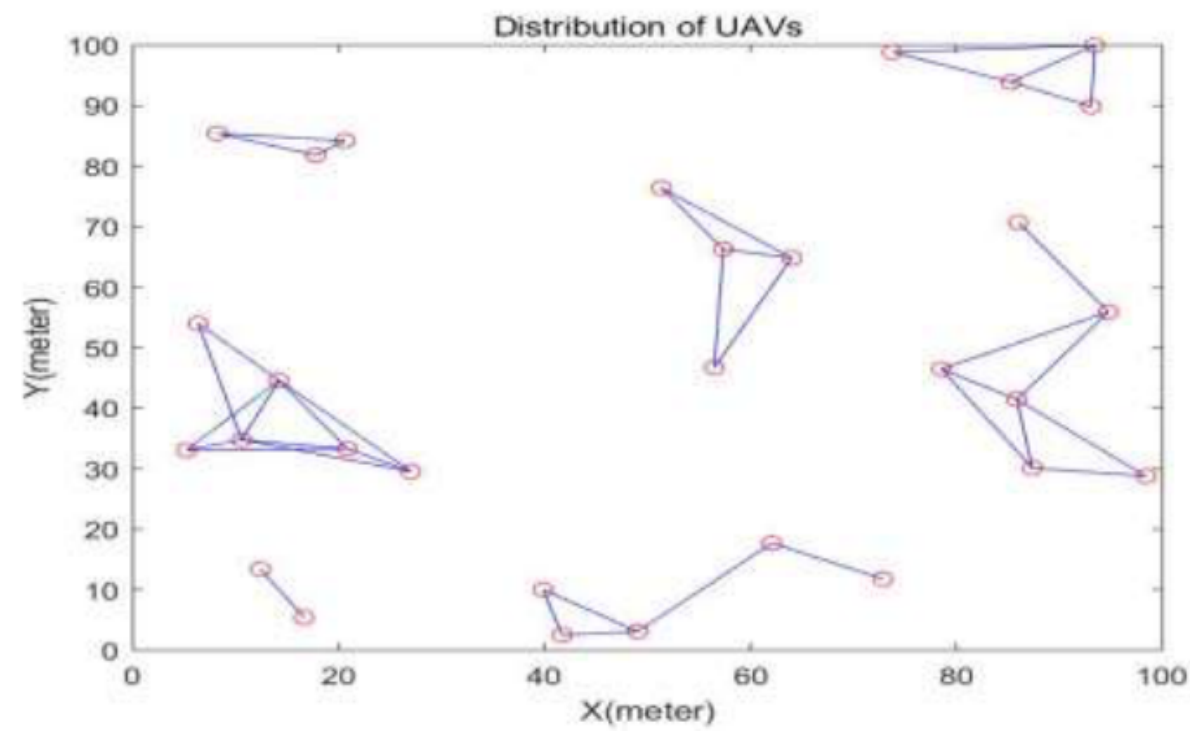

The above figure shows the positions of the UAV drones. The Red circles represent the estimated drone positions, and the blue lines represent the Ultra-Wideband measurements.

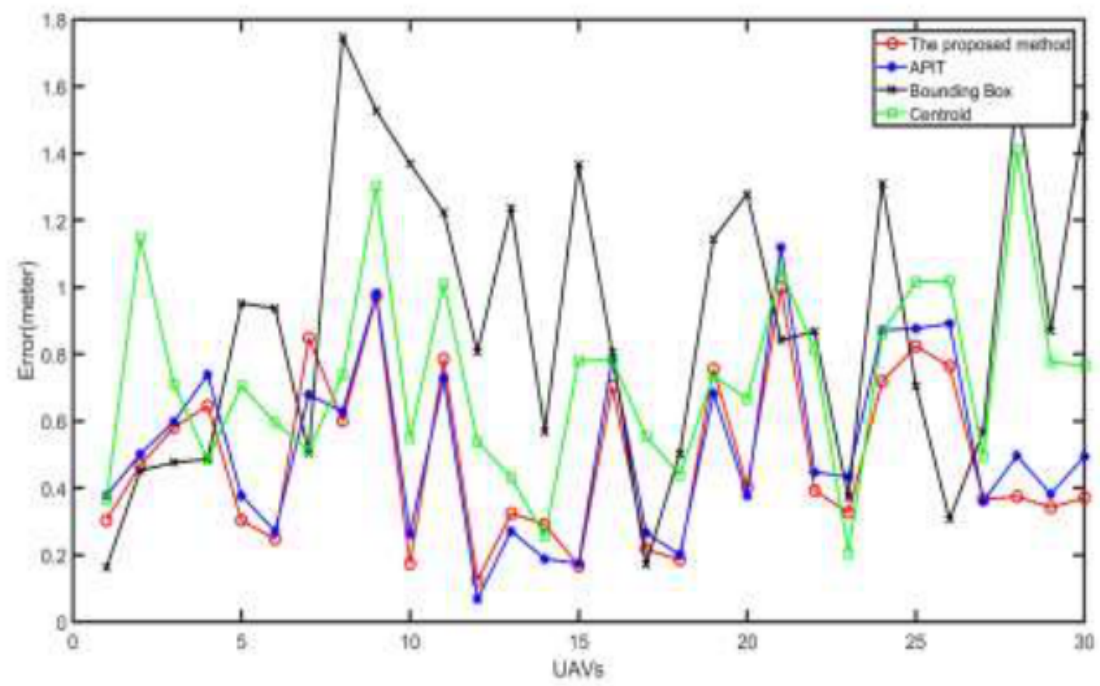

The above graph illustrates the comparisons between our different cooperative localization methods. As clearly shown by the graph, our proposed Double Kalman Filter method outperforms APIT, Bounding Box, and the Centroid methods. 


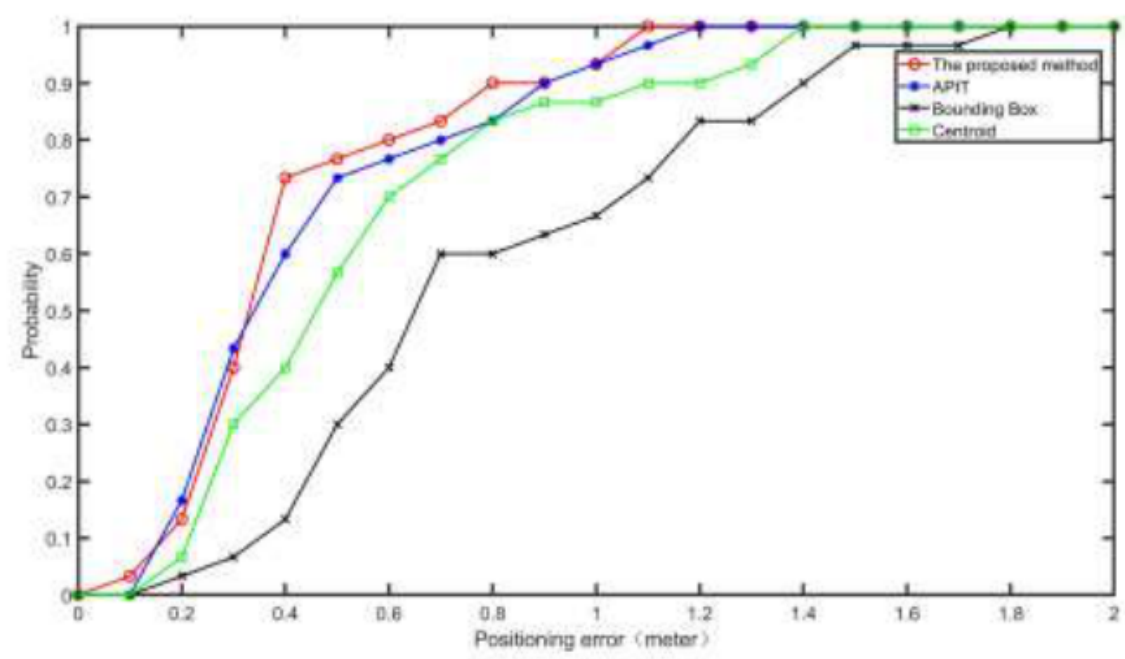

The above figure illustrates the probability of having a certain amount of error. From the graph, it can be shown that the proposed Double Kalman Filter method has a higher probability of having a lower error than the APIT, Bounding Box, and Centroid methods.

\begin{tabular}{|c|c|c|c|c|}
\hline & $\begin{array}{c}\text { The proposed } \\
\text { method }\end{array}$ & APIT & Bounding Box & Centroid \\
\hline Mean Error $(\mathrm{m})$ & 0.40 & 0.43 & 0.54 & 0.67 \\
\hline $95 \%$ Error $(\mathrm{m})$ & 1.11 & 1.24 & 1.37 & 1.45 \\
\hline
\end{tabular}

The table above demonstrates that the error of the proposed Double Kalman Filter method is on average less than that of the APIT, Bounding Box, and Centroid methods. The Mean Error is less, as well as the high end error, as shown in the $95^{\text {th }}$ percentile.

\section{CONCLUSION}

The main contribution of this paper is an algorithm to cooperatively localize nodes in a UAV Swarm. The method fuses Global Position Systems (GPS), Global Positioning System (GPS), Inertial Measurement Unit (IMU) or Inertial Navigation System (INS), and Ultra-Wideband (UWB) using a Double Kalman Filter. The results of our simulation were promising, showing that the new method outperformed APIT, Bounding Box, and Centroid methods.

\section{REFERENCES}

[1] ChungSoonJo, ParanjapeAA, DamesP, et al. A Survey on Aerial Swarm Robotics[J]. IEEE Transactions on Robotics, 2018:1-19.

[2] Marek J, Štěpánek, Ladislav. Accuracy and availability of the satellite navigation system GPS[C]// International Conference on Microwave Techniques. IEEE, 2010.

[3] Huang D. Evidential problems with GPS accuracy: device testing[J]. Auckland University of Technology, 2013.

[4] Macgougan G, O"Keefe K, Klukas R . Ultra-wideband ranging precision and accuracy[J]. Measurement Science and Technology, 2009, 20(9):095105.

[5] He T, Huang C, Blum B M, et al. Range-free localization schemes for large scale sensor networks[C]// International Conference on Mobile Computing \& Networking. 2003.

[6] Bing H C, Vandenberghe L, Yao K. Distributed algorithm for node localization in wireless ad-hoc networks[J]. Acm Transactions on Sensor Networks, 2010, 6(1):1-20. 
[7] Bulusu N, Heidemann J, Estrin D. GPS-less low-cost outdoor localization for very small devices[J]. 2000, 7.

[8] Guler S, Abdelkader M, Shamma J S. Infrastructure-free Localization of Aerial Robots with Ultrawideband Sensors[J]. 2018.

[9] Wan J, Shaocong B U, Zhong L. Cooperative localization algorithm of multi-UAVs based on dynamic hybrid belief propagation[J]. Journal of Beijing University of Aeronautics \& Astronautics, 2016.

[10] Praats B , Berkvens R, Weyn M , et al. Large Scale Distributed Localization Based on RSS and Mass-Spring Model[C]// 2nd International Workshop on Distributed Embedded Systems (DEM 2015). IEEE, 2015.

[11] https://www.systemdynamics.org/what-is-sd 